NASA Contractor Report 198488

AIAA-96-1780

\title{
Refraction and Shielding of Noise in Non-Axisymmetric Jets
}

Abbas Khavaran

NYMA, Inc.

Brook Park, Ohio

May 1996

Prepared for

Lewis Research Center

Under Contract NAS3-27186

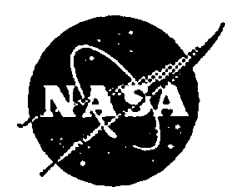

National Aeronautics and

Space Administration 



\title{
Refraction and Shielding of Noise in Non-Axisymmetric Jets
}

\author{
Abbas Khavaran * \\ NYMA, Inc., Lewis Research Group, Cleveland, OH 44135
}

\begin{abstract}
This paper examines the shielding effect of the mean flow and refraction of sound in non-axisymmetric jets. A general three-dimensional ray-acoustic approach is applied. The methodology is independent of the exit geometry and may account for jet spreading and transverse as well as streamwise flow gradients. We assume that noise is dominated by small-scale turbulence. The source correlation terms, as described by the acoustic analogy approach, are simplified and a model is proposed that relates the source strength to $7 / 2$ power of turbulence kinetic energy. Local characteristics of the source such as its strength, time- or length-scale, convection velocity and characteristic frequency are inferred from the mean flow considerations. Compressible Navier Stokes equations are solved with a $\mathrm{k}-\epsilon$ turbulence model. Numerical predictions are presented for a Mach 1.5, aspect ratio 2:1 elliptic jet. The predicted sound pressure level directivity demonstrates favorable agreement with reported data, indicating a relative quiet zone on the side of the major axis of the elliptic jet.
\end{abstract}

$\begin{array}{cl} & \text { Nomenclature } \\ a, b & \text { length of semi major and minor axes } \\ a_{\infty} & \text { freestream sound speed } \\ a(\mathbf{X}) & \text { local sound speed } \\ C(\mathbf{X}) & \text { normalized sound speed } \\ D_{e q} & \text { area equivalent jet diameter } \\ k & \text { turbulent kinetic energy }=\overline{v_{i} v_{i}} / 2 \\ \mathbf{M} & \text { Mach vector } \\ p_{i} & \text { component of phase normal } \mathbf{p} \\ s & \text { arc length } \\ T & \text { turbulence intensity } \overline{v_{i} v_{i}} / 3 \\ \mathbf{U}_{\mathbf{c}} & \text { source convection velocity } \\ U_{j} & \text { jet exit velocity } \\ v_{i} & \text { fluctuating velocity component } \\ \epsilon & \text { dissipation rate of kinetic energy } \\ & =\nu \overline{\left(\partial v_{i} / \partial X_{j}\right)\left(\partial v_{i} / \partial X_{j}\right)} \\ \theta_{\infty} & \text { polar observer angle } \\ \Theta & \text { momentum thickness } \\ \xi_{1}, \xi_{2} & \text { elliptic coordinates } \\ & \end{array}$

\footnotetext{
* Senior Research Engineer, Member AIAA.
}

$\begin{array}{cl}\rho & \text { density } \\ \tau & \text { time-delay of correlation } \\ \phi_{\infty} & \text { azimuthal observer angle } \\ \omega, \Omega & \text { observer and source frequencies } \\ \text { Subscripts } & \\ o & \text { source location } \\ \infty & \text { far field }\end{array}$

\section{Introduction}

The aim of the present study is to determine the far-field directivity of sound due to a jet issued from a non-axisymmetric exit plane. The theoretical argument is a derivative of Lighthill's Acoustic Analogy, with modifications to account for the three-dimensional refraction effects. It is generally accepted that, in theory, direct numerical simulation (DNS) based on the full, compressible Navier-Stokes equations govern both the generation and propagation of sound. Current efforts in computational aeroacoustics ${ }^{1}$ (CAA) are directed towards resolving various computational issues, such as grid resolution (for frequencies of interest), computational domain, boundary conditions, finite difference scheme and so on. The presence of shock expansion fans, under imperfectly expanded conditions, will require additional attention in order to predict the shockassociated noise.

Apart from DNS, other computational models will require some sort of turbulence modeling to predict the mixing noise of a typical shock-free jet. For example, Mankbadi et. $\mathrm{al}^{2,3}$ have opted for an extension of the large-eddy simulation approach (LES). This approach, models the fine-scale turbulence and calculates the large-scale components from the filtered NavierStokes equations. The calculated sound field is thus associated with the large-scale turbulence. Further simplifications were proposed ${ }^{4,5}$ by neglecting viscosity and nonlinear effects utilizing linearized Euler equations (LEE). This last approach is less computer intensive and has been suggested as an alternative to (LES) for three-dimensional geometries. Sample predictions with LES and LEE are presented in the references cited above. The results are encouraging and succeed in capturing the near-field directivity of jet noise at Strouhal 
numbers of interest.

Lighthill's formulations, on the other hand, suggests a source correlation term based primarily on the unsteady velocity fluctuations. Complications associated with numerical calculation of the Lighthill's source term have resulted in various modeling efforts. These efforts have produced alternatives to Lighthill's source term, such as those derived from a mean flow solution and with an appropriate turbulence modeling. Meanwhile, the flow-acoustic interaction is explicitly accounted for as an additional superimposing term.

We start with a brief review of the source modeling as derived from a compressible Navier-Stokes solution with a $k-\epsilon$ turbulence model (e.g., Ref. 6). Our focus, however, is the assessment of the mean flow effects on directivity of jet noise.

Two models are proposed for acoustic predictions. These models differ in the application of flow-acoustic interaction. The first model incorporates Balsa's ${ }^{7}$ solution. Balsa examines the high frequency solution to Lilley's equation in a cylindrical coordinate system. He obtains directivity factors for each of the quadrupoles contained within a turbulent eddy volume element. To extend Balsa's solution to non-axisymmetric jets, we apply a quasi-3D approach. This is done by integrating Balsa's solution azimuthally, i.e., treating each streamwise jet slab as a representative of the entire jet volume. The predicted sound field, though void of any azimuthal directivity, may be argued to account for 3D distribution in source strength as well as the surrounding mean flow.

In the second model, a full $3 \mathrm{D}$ ray-acoustic approach is applied. A system of six propagation equations is solved numerically in a rectangular coordinate system. We map the CFD solution to a uniform grid, i.e., a grid independent of the streamwise direction, and apply a three-dimensional spline interpolation on flow parameters of interest. Mean flow gradients are computed readily from the ensuing spline coefficients as the numerical solution of refraction equations proceeds along rays of geometric acoustics. Although a ray-acoustic solution succeeds in capturing the far-field azimuthal variation in sound, the intensive numerical computations suggest that the methodology should be considered only when the non-axisymmetric exit geometry may result in a relatively significant azimuthal directivity.

The source characteristic is described similarly in both approaches. This includes the simplification of fourth-order space-time correlations ${ }^{6}$ and separation of space and time factors. A new function is proposed for the time-factor of the correlation. This function, re- placing the Gaussian distribution employed in the earlier models ${ }^{11}$, helps improve the predicted spectra.

As an example, a shock-free Mach 1.5 elliptic jet with aspect ratio of $2: 1$ is selected. Aerodynamic predictions are compared with data as well as predictions for a base convergent-divergent round nozzle, operating at similar conditions. The acoustic predictions include sound pressure level directivity (SPL), as well as 1/3octave band spectra.

\section{Method of Solution}

It is generally recognized that at subsonic and lower supersonic speeds, small scale turbulence is the primary acoustic source. Each finite volume of turbulence, may be described as a multipole source that convects downstream and emits sound that is refracted by mean flow gradients. As jets becomes highly supersonic, large-scale structures or instability waves of the flow ${ }^{8}$ become increasingly more active. In the present study, we assume that fine-scale turbulence is the source of jet noise. With a compact eddy approximation, the solution is usually expressed as a Fourier transform of space-time correlation function, integrated over the entire jet volume. Availability of unsteady flow solutions should, in principle, make it possible to compute correlation functions of various order and orientation. Today's computational capabilities, perhaps, should encourage this effort, at least, for a selected number of source volume elements within the jet. The immediate benefit would include assessment of a number of simplifying assumptions that have traditionally been employed for source modeling. For the present work, however, we follow an approach based on separability of space-time correlation and rather focus on refraction and shielding of non-axisymmetric geometries.

\subsection{Source and Spectra}

As described previously, any attempt to integrate the source correlation function over the jet volume requires modeling of these functions. Reference 6 outlines a procedure used in the present study and we briefly discuss some of the more important steps. Roughly speaking, various regions of turbulence of size $\ell$, commonly referred to as an eddy volume element, radiate sound to far-field that may be summed up for all regions of the jet if the correlation volume elements radiate independently. In addition, acoustical compactness which allows for the use of elementary solutions like simple sources to model complex sources of sound such as quadrupoles requires that $\omega \ell / a_{\infty}$ be small. This condition requires that the r.m.s. velocity fluctuation be small relative to ambient sound speed ${ }^{9}$ , i.e., $\left\langle v^{2}\right\rangle^{1 / 2} / a_{\infty} \ll 1$, which makes the turbulence 
length-scale small relative to the acoustic wave length. It should be noted that the compactness condition is not that restrictive and may hold true even at moderate Mach numbers. Assuming that noise is dominated by fine-scale turbulence, contributions from the self-noise term, in absence of refraction and convection, is shown to be due to a fourth-order space-time correlation function $\overline{v_{i} v_{j} v_{k}^{\prime} v_{l}^{\prime}}$. As is usually done, the above term is expressed as a linear combination of second-order correlations $\overline{v_{i} v_{j}^{\prime}}$. This is followed by the space-time separation given as $\overline{v_{i} v_{j}^{\prime}}=R_{i j}(\vec{\zeta}) g(\tau)$, where $\vec{\zeta}$ and $\tau$ are the separation vector and time-delay respectively. For homogeneous isotropic turbulence, Batchelor ${ }^{10}$ suggests a space function of the form

$$
R_{i j}(\vec{\zeta})=T\left[\left(f+\frac{1}{2} \zeta f^{\prime}\right) \delta_{i j}-\frac{1}{2} f^{\prime} \zeta_{i} \zeta_{j} / \zeta\right]
$$

Details of $f(\zeta)$ are provided in Ref. 10 and are not repeated here. A Gaussian function is normally selected for the time-factor part as $g(\tau)=\exp \left(-\tau^{2} / \tau_{o}^{2}\right)$, with $\tau_{0}$ representing the characteristic time-delay of the correlation. It was shown (Ref. 6) that $\tau_{o}$ may be derived from the ratio of kinetic energy of turbulence $k$ and its dissipation rate $\epsilon$ as $1 / \tau_{o} \sim \epsilon / k$. Noise spectra is calculated from the Fourier transform of the autocorrelation function

$$
I(\Omega)=\int_{0}^{\infty} g^{2}(\tau) e^{i \Omega \tau} d \tau
$$

which results in $I(\Omega)=\tau_{0} \sqrt{\pi / 2} e \frac{-\left(\Omega r_{0}\right)^{2}}{8}$. In general function $g(\tau)$ may be written to include other powers of $\tau$. For example function

$$
g(\tau)=e^{-c_{1}\left(\tau / \tau_{0}\right)^{2}-c_{2} \sqrt{c_{3}+\left(\tau / \tau_{0}\right)^{2}}}
$$

with positive constants $c 1, c 2$ and $c 3$ transforms the above integral into a series of modified Bessel functions $K$ and with added empiricism as a result of the new constants. With $c_{3}=0$ and $g(\tau)=e^{-c_{1}\left(\tau / \tau_{0}\right)^{2}-c_{2}\left|\tau / \tau_{0}\right|}$ we have

$$
I(\Omega)=\frac{\tau_{o}}{2 \sqrt{c_{1}}} \sqrt{\pi / 2} \operatorname{Re}\left\{e^{\Upsilon^{2}} \operatorname{erc} f(\Upsilon)\right\}
$$

where

$$
\Upsilon=\frac{c_{2}-i\left(\Omega \tau_{o} / 2\right)}{\sqrt{2 c_{1}}}
$$

and $\operatorname{erc} f(\Upsilon)$ denotes a complementary error function. Constant $c_{2}$ provides additional flexibility to shape the spectra at higher frequencies. With $c_{2}=0$, the solution reduces to that given in Ref. 11. All acoustic predictions presented here are based on equation (4a) with $c_{1}=2.0$ and $c_{2}=1.0 e-5$.
Noise spectra due to a unit volume of turbulence, and arising from the source correlation term $\overline{v_{1} v_{1} v_{1}^{\prime} v_{1}^{\prime}}$ may be written as

$$
I_{1111}(\Omega)=\frac{3}{8 \sqrt{\pi}} \rho_{o}^{2} k^{\frac{\tau}{2}}\left(\Omega \tau_{o}\right)^{4} \frac{1}{2 \sqrt{c_{1}}} \operatorname{Re}\left\{e^{\Upsilon^{2}} \operatorname{ercf}(\Upsilon)\right\}
$$

A Doppler factor relates source frequency $\Omega$ to the observer frequency $\omega$. Contributions from other source correlation components $I_{i j k \ell}$ are expressed similarly ${ }^{6}$.

\subsection{Flow-Acoustic Interaction}

It is known that the operator part of the Lilley's equation accounts for refraction as well as convection. A close-form solution to this equation was given by $\mathrm{Balsa}^{7}$ in the high frequency limit and in a cylindrical coordinate system. Although Balsa's directivity factors are limited to axisymmetric jets, the solution may be integrated azimuthally for various streamwise jet slices to predict the SPL directivity for non-axisymmetric flows. This type of prediction, referred to as a quasi-3D solution, though void of any azimuthal variation in sound, is very robust and should be considered a first approximation to noise prediction for arbitrary geometries when far-field azimuthal directivity is relatively small.

The general directivity of sound for jets of arbitrary geometry may be assessed using ray-acoustics in a high frequency limit. In a high frequency approximation, it is normally assumed that the acoustic wave length is shorter than the characteristic length of the mean flow. For high speed jets, this approximation can be used effectively to predict the more energetic part of the sound spectra and for Helmholtz numbers $\left(f D / a_{\infty}\right)$ as small as one (see Tester and Morfey ${ }^{12}$ ). When a parallel flow approximation is invoked, refraction of jet noise through a sheared mean flow of arbitrary geometry may be formulated as a two-dimensional geometric acoustic problem ${ }^{13}$. It is argued ${ }^{14}$ that for high speed flows, jet spreading may play a significant role in defining the size of cone of silence which is formed in the neighborhood of the downstream jet axis. In such cases, flow spreading may be accounted for by formulating a three-dimensional ray-acoustic problem. Thus, as acoustic energy, released from each finite volume of turbulence propagates out of the shear-layer, its spreading is inferred from the area of a ray-tube surrounding that ray.

For an inviscid flow governed by linearized gas dynamic equations, the coordinate $\mathbf{X}$ of the emitted sound at any point $s$ along the ray is determined from a set of six equations ${ }^{14,15}$

$$
\frac{d X_{i}}{d s}=T_{i j} p_{j}+M_{i} / C \quad i, j=1,2,3
$$




$$
\frac{d p_{i}}{d s}=-\frac{1}{2} p_{j} \frac{\partial T_{j k}}{\partial X_{i}} p_{k}-p_{j} \frac{\partial}{\partial X_{i}}\left(\frac{M_{j}}{C}\right)+\frac{1}{2} \frac{\partial}{\partial X_{i}}\left(C^{-2}\right)
$$

and

$$
\begin{gathered}
T_{i j}=\delta_{i j}-M_{i} M_{j} \\
\mathbf{M}(\mathbf{X})=\frac{\mathbf{U}(\mathbf{X})}{a(\mathbf{X})}, \quad C(\mathbf{X})=\frac{a(\mathbf{X})}{a_{\infty}} .
\end{gathered}
$$

Vector $\mathrm{p}$ is normal to the phase front and $M_{i}$ denotes the component of the Mach vector $M$. Equations (6) are solved numerically subject to initial conditions associated with the source location and direction of emission. Let unit vector $\hat{\mathbf{X}}=(\cos \mu, \sin \mu \cos \delta, \sin \mu \sin \delta)$ denote the direction of emission at the source, and subscripts $o$ and $\infty$ refer to source and observer locations respectively, then

$$
\begin{gathered}
\mathbf{X}=\mathbf{X}_{\mathbf{o}} \quad \text { at } \quad s=0 \\
\mathbf{p}_{o}=\lambda \hat{\mathbf{X}}+\left(\lambda \hat{\mathbf{X}} \cdot \mathbf{M}-\frac{1}{C}\right) \frac{\mathbf{M}}{\beta^{2}} \\
\lambda=\left[\beta^{2}+(\hat{\mathbf{X}} \cdot \mathbf{M})^{2}\right]^{-\frac{1}{2}} / C, \quad \beta^{2}=1-|\mathbf{M}|^{2} .
\end{gathered}
$$

As rays emerge from the shear layer, they become straight and the ray speed $\lambda$ approaches one. For every set of radiation angles $(\mu, \delta)$ a pair of angles $\left(\theta_{\infty}, \phi_{\infty}\right)$ may be calculated from equations (6). Variation of the ray-tube area is then expressed as the Jacobian of the transformation, i.e., $\left|\partial\left(\theta_{\infty}, \phi_{\infty}\right) / \partial(\mu, \delta)\right|$. It is shown ${ }^{14,16}$ that the far-field mean square pressure directivity for a convecting quadrupole source of strength $Q_{i j}(\Omega)$ becomes

$$
\begin{gathered}
\overline{P_{\mathcal{Q}_{i j}}^{2}} \propto\left(\frac{\rho_{\infty} a_{\infty}}{4 \pi R}\right)^{2} \Omega^{4}\left(\frac{Q_{i j} p_{i} p_{j}}{|\mathbf{p}|^{2}}\right)_{0}^{2}\left(\frac{a_{o}^{2}}{a_{\infty}^{2}} \frac{\lambda_{o}^{3}}{\rho_{o}}\right) \times \\
\frac{\left(1-\frac{\mathbf{U}_{o}}{a_{\infty}} \cdot \mathbf{p}_{o}\right)^{2}\left(1-\frac{\mathbf{U}_{\infty}}{a_{\infty}} \cdot \mathbf{p}_{\infty}\right)^{2}}{\left(1-\frac{\mathrm{U}_{c}}{a_{\infty}} \cdot \mathbf{p}_{\mathbf{o}}\right)^{5}}\left(\frac{\sin \mu}{\sin \theta_{\infty}} \frac{1}{\left|\frac{\partial\left(\theta_{\infty}, \phi_{\infty}\right)}{\partial(\mu, \delta)}\right|}\right)
\end{gathered}
$$

where $\mathbf{U}_{\mathbf{c}}$ is the source convection velocity and $\omega$ is the observer frequency related to source frequency $\Omega$ as

$$
\omega=\Omega /\left(1-\frac{\mathrm{U}_{\mathrm{c}}}{a_{\infty}} \cdot \mathbf{p}_{\mathbf{o}}\right)
$$

For an isotropic quadrupole source, $Q_{i j} p_{i} p_{j}=Q_{0}|\mathbf{p}|^{2}$ If source spectral power density $Q_{0}^{2}$ is replaced with $I_{1111} / \Omega^{4}$ from (5), the directivity pattern is found as

$$
\begin{aligned}
& \overline{P_{\mathcal{Q}_{0}}^{2}} \propto\left(\frac{a_{\infty}}{4 \pi R}\right)^{2}\left(\rho_{\infty}^{2} k^{\frac{T}{2}}\right)\left(\Omega \tau_{0}\right)^{4} R e\left\{e^{\Upsilon^{2}} \operatorname{ercf}(\Upsilon)\right\}\left(\frac{a_{o}^{2}}{a_{\infty}^{2}} \lambda_{o}^{3} \rho_{o}\right) \\
& \times \frac{\left(1-\frac{\mathrm{U}_{0}}{a_{\infty}} \cdot \mathbf{p}_{o}\right)^{2}\left(1-\frac{\mathrm{U}_{\infty}}{a_{\infty}} \cdot \mathbf{p}_{\infty}\right)^{2}}{\left(1-\frac{\mathrm{U}_{c}}{a_{\infty}} \cdot \mathbf{p}_{0}\right)^{5}}\left(\frac{\sin \mu}{\sin \theta_{\infty}} \frac{1}{\left|\frac{\partial\left(\theta_{\infty}, \phi_{\infty}\right)}{\partial(\mu, \delta)}\right|}\right) .
\end{aligned}
$$

Roughly speaking, when the difference between $\mathbf{U}_{\mathbf{o}}$ and $\mathbf{U}_{\mathbf{c}}$ is small, and assuming that $\mathbf{U}_{\infty}$ is negligible, the directivity pattern scales as $\left(1-\frac{U_{c}}{a_{\infty}} \cdot p_{o}\right)^{-3}$ outside the zone of silence. Near and within the boundary of zone of silence, the factor $\left(\frac{\sin \mu}{\sin \theta_{\infty}}\right) /\left|\partial\left(\theta_{\infty}, \phi_{\infty}\right) / \partial(\mu, \delta)\right|$ al ters the above directivity and results in concentration of noise near the boundary of zone of silence, i.e., a sharp peak followed by a rapid decay into the zone of silence.

\subsection{Aerodynamic Predictions}

Flow predictions were made with the NPARC Navier-Stokes code and with a recently installed ${ }^{17}$ low Reynolds number $\mathrm{k}-\epsilon$ model of Chien ${ }^{18}$. The conical and elliptic jets considered had an exit area of 1.571 in $^{2}$ each, with aspect ratio of $\mathrm{AR}=2: 1$ for the elliptic jet. Both nozzles are operated at the design pressure ratio of 3.67 and $564^{\circ} \mathrm{R}$ total temperature to give exit Mach number of 1.5. Some aerodynamic and acoustic data $^{19}$ were available for validation. Because of symmetry, only one quarter of the elliptic jet is modeled. The major and minor axes are planes of symmetry modeled with NPARC's slip wall boundary conditions. A grid having $121 \times 91$ points in axial and radial directions and 25 points in circumferential direction was selected. The domain of the grid includes 10 diameters into the nozzle and up to 70 diameters downstream of the exit plane.

For convenience, elliptic coordinates $\xi_{1}$ and $\xi_{2}$ are introduced

$$
y=\xi_{1} \cos \xi_{2}, \quad z=\left(\xi_{1} / p\right) \sin \xi_{2}, \quad x=x,
$$

where $x$ denotes the streamwise direction. The above coordinates maintain a constant aspect ratio of $p=a / b$, with an area element $d A=\left(\xi_{1} / p\right) d \xi_{1} d \xi_{2}$ (see figure 1a). Within one quarter of the elliptic jet, the azimuthal angle $\phi$, measured from the major axis, is equally divided over 25 grid points and relates to elliptic angle $\xi_{2}$ as $\tan \xi_{2}=p \tan \phi$. Two-dimensional views of the elliptic grid for major axis plane $x y$ and spanwise plane $y z$ are shown in figures $1 \mathrm{~b}$ and $1 \mathrm{c}$.

The centerline velocity decay for both $2 \mathrm{D}$ and 3D flow predictions are sensitive to the selection of the spanwise boundary conditions. With a free-type boundary condition, the predicted centerline velocity decay (figure 2) shows a supersonic core length of $11.9 \mathrm{D}$ and $11.3 D_{e q}$ for round and elliptic jets compared to $14.2 D$ and $11.2 D_{e q}$ reported experimentally ${ }^{19}$. Although a slip-type boundary condition will improve the $2 \mathrm{D}$ predictions (see Ref. 11), in the interest of $3 \mathrm{D}$ acoustic predictions, however, boundary conditions favorable to elliptic jet are selected for mean flow computations. 
Velocity profiles along the major and minor axes of the elliptic jet are shown in figures $3 \mathrm{a}$ and $3 \mathrm{~b}$ respectively. Comparison with data of Seiner ${ }^{19}$ demonstrates good agreement along the minor axis plane. The major axis predictions, on the other hand, appear to underestimate the spreading rate. Similar conclusions were drawn from the axial distribution of momentum thickness defined for compressible flows

$$
\Theta=\int_{0}^{\infty} \frac{\rho U}{(\rho U)_{c l}}\left(1-\frac{U}{U_{c l}}\right) d r
$$

where subscript $c l$ denotes the centerline values. The predicted momentum thickness (figure 4a) shows little difference along the major and minor axes near the exit. A more pronounced difference is observed downstream where thickness along the major axis shows a slightly faster growth rate. The round nozzle momentum thickness in figure $4 \mathrm{a}$ is in agreement with the elliptic jet's minor axis. Data of figure $4 \mathrm{~b}$ indicates that momentum thickness is nearly equal along both axes over the length of the potential core and starts to grow at a faster rate along the major axis near the end of the core. Notice that the round nozzle momentum thickness, shown here for a design Mach number of 2.0 , is in agreement with the general behavior along the minor axis as was concluded from predictions of figure $4 \mathrm{a}$.

Predicted turbulence intensity profiles along major and minor axes of the elliptic jet are shown in figures $5 \mathrm{a}$ and $5 \mathrm{~b}$. Here the percent turbulence is defined as $100 \times\left(v_{i} v_{i} / 3\right)^{1 / 2} / U_{j}$. Both figures indicate a maximum level in the neighborhood of the jet lip-line and an exit value of $11.3 \%$, that will gradually increase to $12.5 \%$ at about $5 D_{e q}$ and decays farther downstream. The centerline value is zero within the core to about $6 D_{e q}$ and will rise to $3.4 \%$ at $7.3 D_{e q}$ and $8.7 \%$ at $10.7 D_{e q}$. Although the existing modifications ${ }^{17}$ in NPARC allow for turbulence kinetic energy input as an inflow boundary condition, the predictions within the core remain insensitive to the specified inflow values. Experiments should be performed to verify turbulence predictions.

\subsection{Acoustic Predictions}

A quasi-3D prediction model may be employed to predict the noise of $3 \mathrm{D}$ jets when the azimuthal directivity is of less significance. This is done by integrating Balsa's solution azimuthally, i.e., treating each streamwise jet slab as a representative of the entire jet. The approach is numerically robust and should be considered as a viable solution when azimuthal directivity is not a design criteria. Geometries such as round lobedmixer nozzles may not result in a significant azimuthal directivity in the far-field. This is more so with increasing the number of lobes.
Sample prediction for Mach 1.5 elliptic jet using a quasi-3D approach will be discussed shortly. A full 3D ray-acoustic solution, on the other hand, is obtained according to equation (10) and in conjunction with equations (6) and (7). Here, as in the 2D case, source strength $k^{7 / 2}$ and characteristic time-scale $\tau_{0}$ are derived from CFD- $k \epsilon$ solutions according to $1 / \tau_{0}=\alpha(\epsilon / k)$. Empirically constant $\alpha$ is included in constant $c_{1}$. Source convection velocity $U_{c}$ is expressed as weighted average of exit and local velocities $\mathbf{U}_{\mathbf{c}}=0.5 \mathbf{U}_{\mathrm{o}}+0.3 \mathbf{U}_{\mathbf{j}}$. For an observer frequency $\omega$ , the corresponding source frequency $\Omega$ is calculated from equation (9), where po denotes the normal to the phase front at the source. At selected observer coordinates $\left(R, \theta_{\infty}, \phi_{\infty}\right)$, the source radiation angles $(\mu, \delta)$ and the corresponding phase normal $p_{0}$ are found by solving (6) and (7) numerically as a boundary-value problem. Once the missing initial values are found, they may be used as a first guess for the neighboring source volume element $\Delta V$ and the process continues.

In order to integrate the propagation equations, a B-spline interpolation of the CFD solutions is carried out in three dimensions

$$
\begin{gathered}
\sum_{\ell=1}^{N_{\xi_{2}}} \sum_{m=1}^{N_{\xi_{1}}} \sum_{n=1}^{N_{x}} C_{n m \ell} B_{n, k_{x}, t_{x}}(x) B_{m, k_{\xi_{1}}, \ell_{\ell_{1}}}\left(\xi_{1}\right) \times \\
B_{\ell, k_{\xi_{2}}, t_{\ell_{2}}}\left(\xi_{2}\right)=f\left(x, \xi_{1}, \xi_{2}\right) .
\end{gathered}
$$

Tensor coefficients $C_{n m \ell}$ may be found for each flow parameter of interest by solving (13) as a system of simultaneous equations applied to data points $\left(x, \xi_{1}, \xi_{2}, f\right)$. Here $k_{x}, k_{\xi_{2}}$ and $k_{\xi_{2}}$ are the orders of spline, $t_{x}, t_{\xi_{1}}$ and $t_{\xi_{2}}$ are the corresponding knot sequence in $x, \xi_{1}$ and $\xi_{2}$ directions respectively ${ }^{23}$, and $B_{n, k, t}$ denotes the $\mathrm{n}$-th $\mathrm{B}$-spline of order $\mathrm{k}$ with respect to knot sequence $t$. It should be noted that the above process assumes a uniform grid, i.e., one independent of the streamwise direction. To this end, a postprocessing of the CFD solution may be necessary. This is usually done by selecting a common spanwise grid structure, say one defined at $X / D_{e q}=10.0$, and consequently mapping the flow field to this grid at each streamwise location using a two-dimensional B-spline interpolation. Once spline coefficients $C_{n m \ell}$ in equation (13) are determined, the directional derivatives become readily available at any point within the jet.

It is known that refraction results in a zone of silence for the high frequency noise. The zone of silence, denoted as $\theta^{\star}\left(\mathbf{X}_{\mathbf{0}}\right)$, and measured from the jet axis, is defined as the smallest polar angle that may receive acoustic signal from a source. This angle is found at each source location $\mathbf{X}_{\mathbf{0}}$ by solving equations (6) subjet to initial conditions $(\mu, \delta)=(0,0)$. Obviously, for 
off-axis sources, a complete refraction may be achieved with a small value of cone angle $\mu$ if $\delta$ is such that sound is directed towards the centerline. This may explain the observed smooth transition of SPL directivity into the zone of silence. However, the peak directivity level for a selected source is in the very neighborhood of $\theta^{\star}$.

Shown in figure 6 is the boundary of zone of silence for sources located on the major axis plane, $x-y$, of the elliptic jet. The source axial location is indicated as the parameter $X_{o} / D_{e q}$. It is clearly seen that for the most part, more energetic segments of the jet, i.e. sources near the lip-line $Y_{o} / a=1.0$, radiate primarily in direction of $\theta^{\star}=50^{\circ}$. This is consistent with the reported directivity for the high Strouhal number noise for Mach 1.5 elliptic jet. Sensitivity of the zone of silence with respect to azimuthal source location is shown in figure 7. Generally speaking, the flow asymmetry will result in an increase in the size of cone of silence as the source moves azimuthally closer the minor axis.

Sound pressure level directivity for elliptic jet as well as Mach 1.5 conical jet are shown in figure 8. Predictions are on a 12 foot sideline. Here, the elliptic jet is treated with the quasi-3D approach. This type of prediction, as indicated earlier, does not produce an azimuthal directivity. Also shown in this figure is the measured SPL directivity ${ }^{19}$. Noise measurements of non-axisymmetric flows, normally show lower noise on the deep side of a jet, such as the major axis of the elliptic jet. In addition, asymmetric jets usually generate less noise compared to conical jets operating under similar conditions. This may be due a combination of factors such as turbulence level, enhanced mixing, and decay in supersonic core length. No data was available for the conical nozzle at this point, however, based on measurements reported at higher temperatures ${ }^{24}$, it is anticipated that the conical nozzle is at least as noisy as the minor axis side of the elliptic jet. Predictions of figure 8 indicate a reasonably good agreement with data in the peak noise level, though the size of the zone of silence seems somewhat exaggerated. Predicted spectra for the two jets are shown in figures 9 and 10 .

We apply a three-dimensional geometric acoustic (3DGA) approach to assess the general directivity of sound for the elliptic jet. A matrix of five polar angles $\theta_{\infty}=50^{\circ}$ to $110^{\circ}$, in increments of $15^{\circ}$, and four azimuthal angles $\phi_{\infty}=0^{\circ}$ to $90^{\circ}$, increments of $30^{\circ}$ is selected.

Shown in figures $11 \mathrm{a}$ and $11 \mathrm{~b}$ are the HFGA spectral predictions along the major axis side of the elliptic jet. Spectral measurements, converted to $1 / 3$-octave band, as well as the quasi-3D predictions are shown in these figures. Data shows some shift in the spectral peak to higher frequencies with increasing polar angle $\theta_{\infty}$. The prediction methods do not exhibit a similar shift as data. It is conjectured that this may be associated with simplistic replacement of the fourth-order space-time correlation functions.

Azimuthal directivity (figure 12), for the most part, shows $2 \mathrm{~dB}$ lower noise along the major axis side, in good agreement with data of figure 8 . Due to the immense numerical calculations associated with rayacoustic predictions, the volume integration was limited to the more energetic elements within the jet. This might reflect as several $\mathrm{dB}$ difference in the noise level as seen in figure 13. In addition, predicted zone of silence appears much larger than measurements (figure 13). Arguably, this may relate to the absence of a frequency-dependent factor in the first term of the expansion series ${ }^{15}$ selected for the GA predictions. The directivity factors suggetsted by Balsa, though obtained in a high frequency limit, contain a wave number factor. It is plausible that addition of the second term in the expansion series could improve the ray-acoustic predictions, in particular near the jet axis where low frequency noise dominates. An accurate prediction of the mean flow profiles and jet spreading may also have a significant bearing on refraction angles. For example, it was shown (Ref. 11 ) that an ambient Mach number of $M_{\infty}=0.1$, will reduce the size of the zone of silence by more that 8 degrees.

The significance of constant $c_{2}$, as used in the the present model for the time-delay function (see Eq. 3) is shown in figure 14. It is seen that the predicted spectra show improvements relative to case when $c_{2}=0.0$.

Figure 15 demonstrates the spherical directivity for a typical jet segment at $4.3<X / D_{e q}<5.8$. This figure suggests that the basic azimuthal pattern of jet noise may be captured readily by selection of appropriate source elements within acoustically active regions of the flow and without a need for complete volume integration.

\section{Concluding Remarks}

We presented a methodology for prediction of jet mixing noise due to noncircular jets. A shock-free elliptic jet with an aspect ratio of $A R=2: 1$ was considered. Predictions assume that noise is generated by small scales of motion and is dominated by the high end of the spectra.

Two prediction models were proposed. A quasi-3D methodology, based on Balsa's solution, should be considered when the azimuthal directivity is of less significance. This approach accounts for asymmetry in source distribution as well as the surrounding mean flow, however, the predicted directivity is axisymmetric. A full $3 D$ ray-acoustic solution, derived from the first term in 
the expansion series, generates an azimuthal directivity compatible with data while the predicted zone of silence remains larger than measurements.

Contributions from large-scale coherent structures may be accounted for via a linear inviscid stability analysis. Stability type analysis is highly geometry dependent and has been fully explored in Ref. 22 for an elliptic geometry assuming a simple representation of the mean velocity profiles.

As discussed, a revisit of the turbulence correlation function for the purpose of source modeling and validation is appropriate at this point. Despite this needed improvement, ray-acoustic approach as presented here, sheds light on the importance of the mean flow interaction and the role it plays in shaping the directivity of jet noise.

\section{Acknowledgements}

This work was supported by NASA Lewis Research Contract NAS3-27186. The technical monitor was E. A. Krejsa. The author is grateful to Dr. J.M. Seiner from NASA Langley for supplying the experimental data.

\section{References}

${ }^{1}$ Tam, C. K. W. and Burton, D. E., "Sound Generated by Instability Waves of Supersonic Flows. Part 2. Axisymmetric Jets," J. Fluid Mech., 138, 1984, pp. 273-295.

${ }^{2}$ Mankbadi, R. R., Hayder, M. E. and Povinelli, L. A., "Structure of Supersonic Jet Flow and its Radiated Sound," AIAA Journal, 32, 1994, pp. 897-906.

${ }^{3}$ Mankbadi, R. R., Shih, S. H., Hixon, R., and Povinelli, L. A., "Direct Computation of Acoustic and Flow Field of a Supersonic Jet Using Large-Eddy Simulation," AIAA Paper 95-0680, 1995.

${ }^{4}$ Mankbadi, R. R., Hixon, R., Shih, S. H., and Povinelli, L. A., "On the Use of Linearized Euler Equations in the Prediction of Jet Noise," AIAA Paper 95$0505,1995$.

${ }^{5}$ Hixon, R., Shih, S. H., and Mankbadi, R. R., "Effect of Input Disturbance on Linearized Euler Equation Prediction of Jet Noise," AIA A Paper 96-0752, 1996.

${ }^{6}$ Khavaran, A., Krejsa, E. A., and Kim, C. M., "Computation of Supersonic Jet Mixing Noise for an Axisymmetric Convergent-Divergent Nozzle," AIAA J. Aircraft, 31, 1994, pp. 603-609.

${ }^{7}$ Balsa, T. F., "The Far Field of High Frequency Convected Singularities in Sheared Flows, with an Application to Jet Noise Prediction," J. Fluid Mech., 74, 1976, pp. 193-208.

${ }^{8}$ Tam, C. K. W., "Aerodynamics of Flight Vehicles: Theory and Practice," NASA Ref. Pub. 1258, Vol. 1, 1991, pp. 311-390.
${ }^{9}$ Lighthill, M. J., "Jet Noise. The Wright Brothers' Lecture," AIAA Journal, 1, 1963, pp. 1507-1517.

${ }^{10}$ Batchelor, G. K. The theory of Homogeneous Turbulence, Cambridge Univ. Press, 1953.

${ }^{11}$ Khavaran, A., and Georgiadis, N. J., "Aeroacoustics of Supersonic Elliptic Jets," AIAA Paper 96-0641, 1996.

${ }^{12}$ Tester, B. J. and Morfey, C. L., "Developments in Jet Noise Modeling - Theoretical Predictions and Comparisons with Measured Data," J. Sound Vib., 46(1), 1976, pp. 79-103.

${ }^{13}$ Goldstein, M. E., "High Frequency Sound Emission from Moving Point Multipole Sources Embedded in Arbitrary Transversely Sheared Mean Flows," J. Sound Vib.,80(4), 1982, pp. 499-522.

${ }^{14}$ Khavaran, A., and Krejsa, E. A., "Refraction of High Frequency Jet Noise in an Arbitrary Jet Flow," AIAA Paper 94-0139, 1994.

${ }^{15}$ Durbin, P. A., "High Frequency Green Function for Aerodynamic Noise in Moving Media, part I: General Theory," J. Sound Vib., 91(4), 1983, pp. 519-525.

${ }^{16}$ Durbin, P. A., "High Frequency Green Function for Aerodynamic Noise in Moving Media, part II: Noise from a Spreading Jet," J. Sound Vib., 91(4), 1983, pp. 527-538.

${ }^{17}$ Georgiadis, N. J., Chitsomboon, T., and Zhu, J. "Modification of the Two-Equation Turbulence Model in NPARC to a Chien Low Reynolds Number k- $\epsilon$ Formulation," NASA TM-106710, 1994.

${ }^{18}$ Chien, K. Y., "Prediction of Channel and Boundary Layer Flows with a Low Reynolds-Number Turbulence Model," AIAA Journal, 20(1), 1982, pp. 33-38.

${ }^{19}$ Seiner, J.M., "Fluid dynamics and Noise Emission Associated with Supersonic Jets," Studies in Turbulence, edited by T.B.Getski, S. Sarkar and G. Speziale, Springer-Verlag, New York, 1992, pp. 297-323.

${ }^{20} \mathrm{Ho}, \mathrm{C} . \mathrm{M}$. and Gutmark, E., "Vortex Induction and Mass Entrainment in a Small Aspect Ratio Elliptic Jet," J. Fluid Mech., 179, 1987, pp. 383-405.

${ }^{21}$ Bridges, J.E. and Hussain, A.K.M.F., "Roles of Initial Condition and Vortex Pairing," J. Sound Vib., 117, part 2., 1987, pp. 289-311.

${ }^{22}$ Morris, P. J., and Bhat, T. R. S., "The Prediction of Noise Radiation from Supersonic Elliptic Jets," AGARD 78th Specialist Meeting on Combat Aircraft Noise, Bonn, Germany, Oct. 1991.

${ }^{23} \mathrm{de}$ Boor, C., A Practical Guide to Splines, SpringerVerlag, New York, 1978

${ }^{24}$ Seiner, J. M., and Ponton, M. K., "Supersonic Acoustic Source Mechanisms for Free Jets of Various Geometries," AGARD 78th Specialist Meeting on Combat Aircraft Noise, Bonn, Germany, Oct. 1991. 


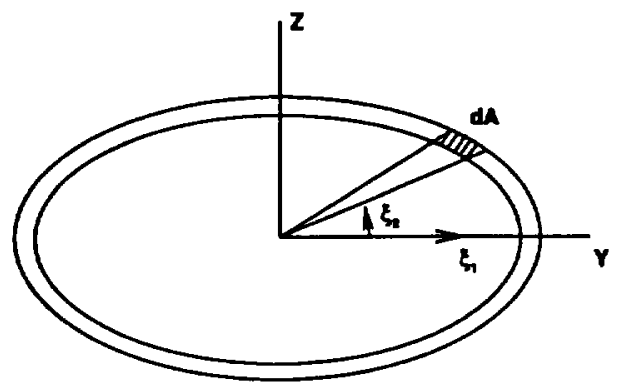

Figure 1a. Elliptic coordinates

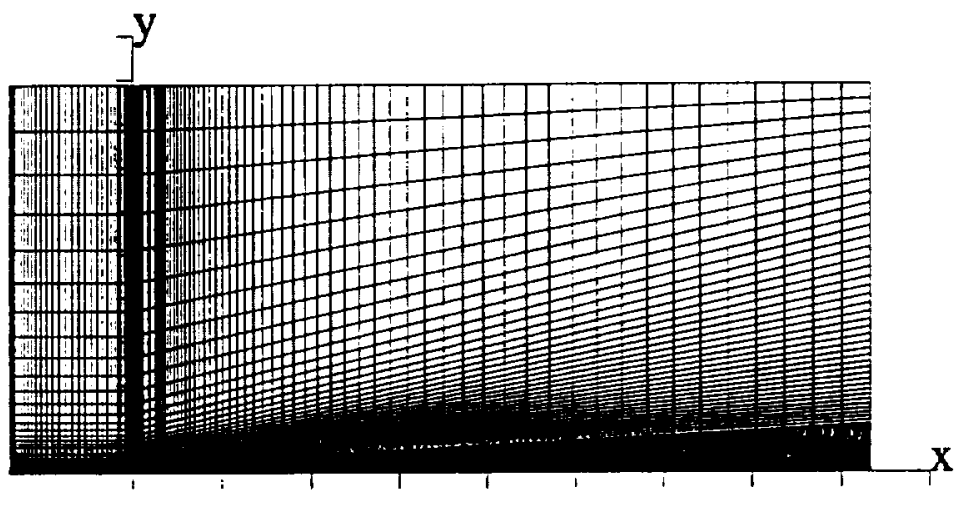

Figure 1b. Grid in major axis plane, jet exit is at $X=0.0$.

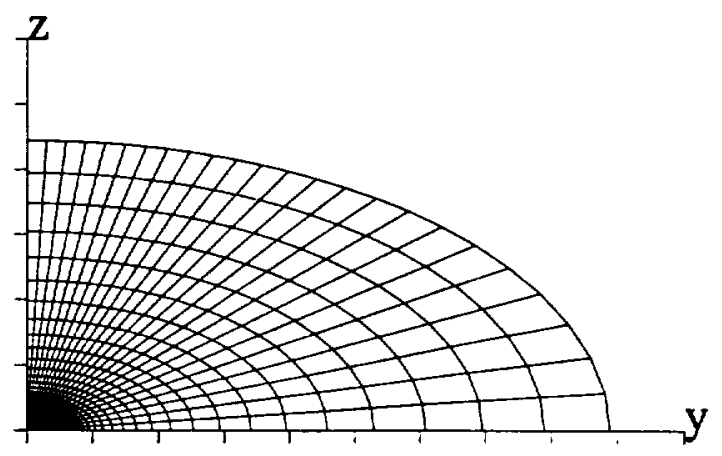

Figure 1c. Grid in a spanwise plane at $X / D_{\odot q}=5.4$.

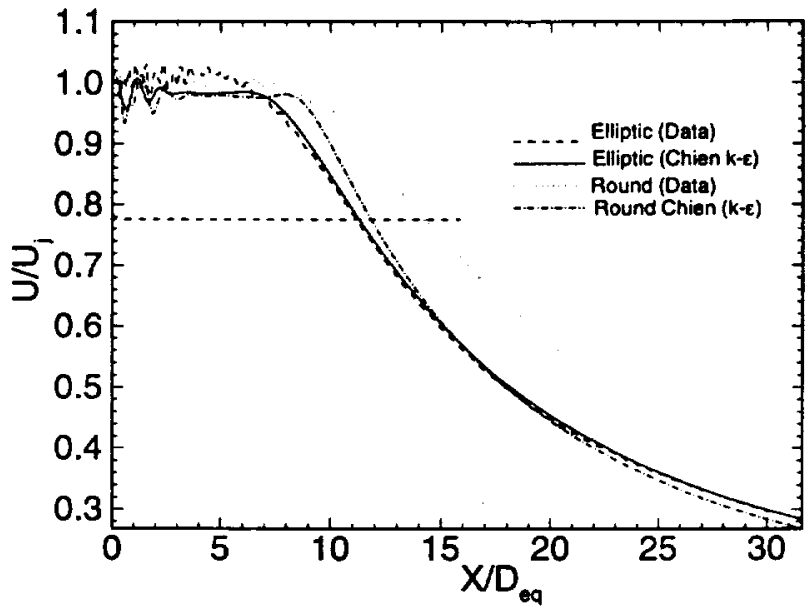

Figure 2. Centerline velocity decay for the elliptic jet and the equivalent $M=1.5$ conical jet. 

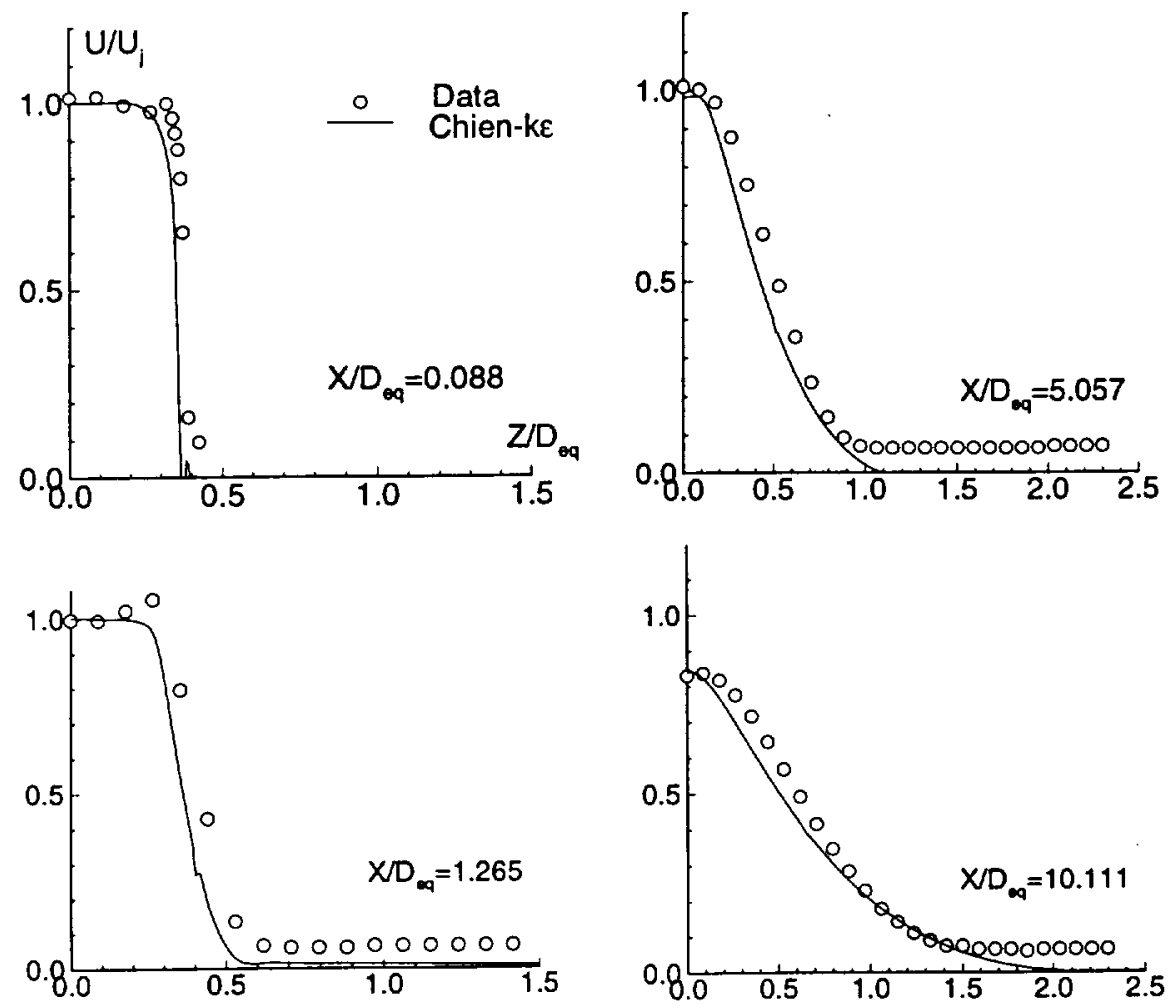

Figure 3a. Velocity profile along the minor axis of the elliptic jet.
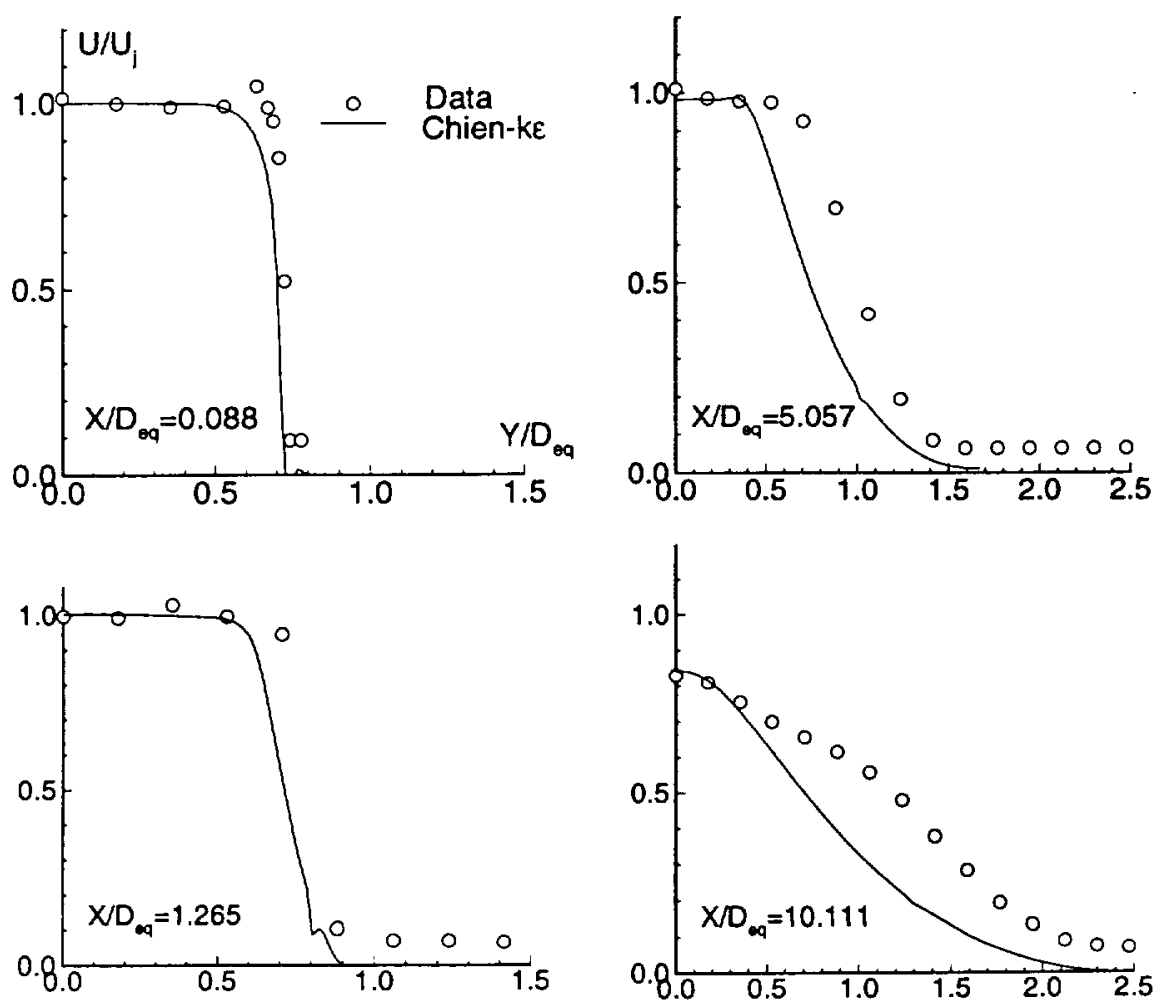

Figure 3b. Velocity profile along the major axis of the elliptic jet. (NPR=3.67, $T=104^{\circ} \mathrm{F}, \mathrm{AR}=2: 1, \mathrm{M}_{\infty}=0.0$ ) 


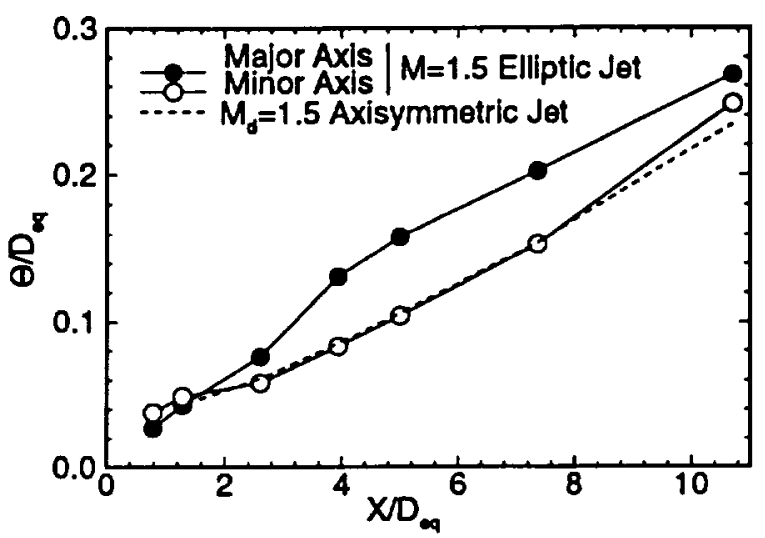

Figure 4a. Predicted momentum thickness for round and elliptic jets ( NPARC-kE).

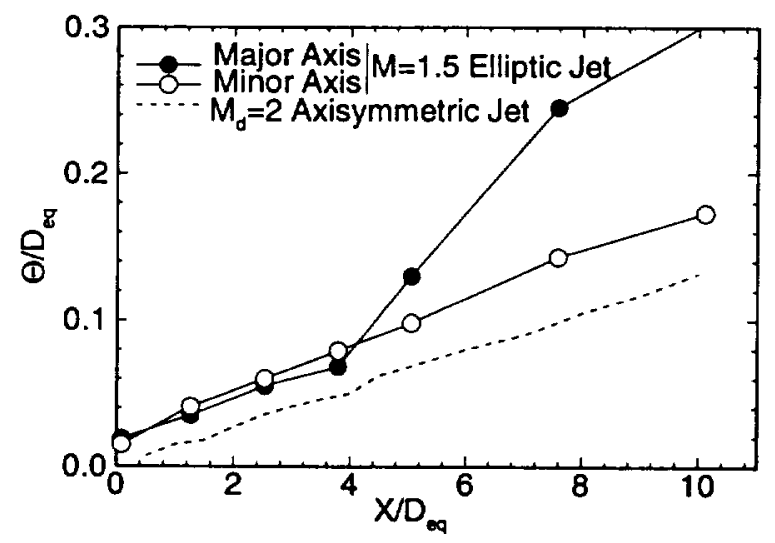

Figure 4b. Measured momentum thickness for round and elliptlc jets (data Ref. 19).

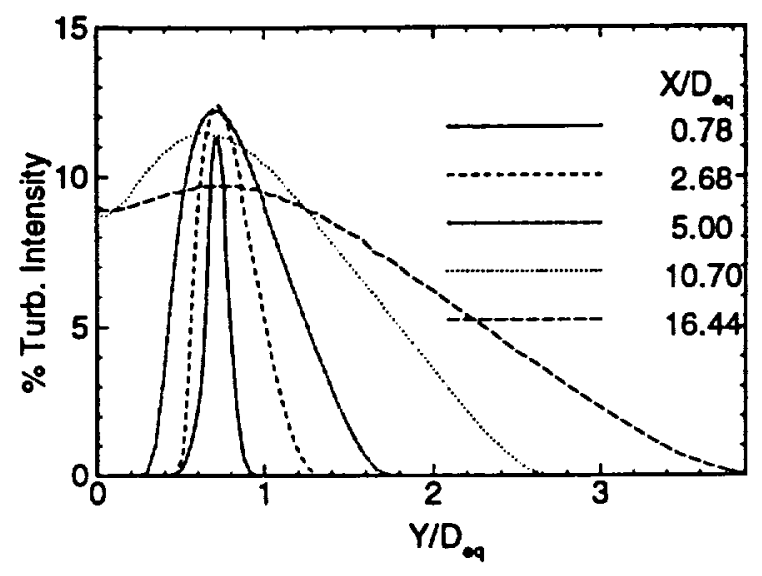

Figure 5a. Predlcted turbulence intensity along the major axis of Mach 1.5 elliptic jet.

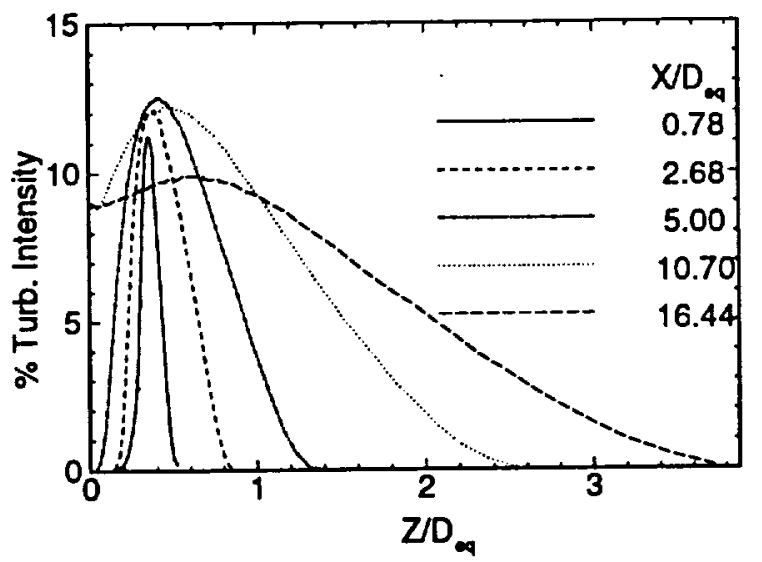

Figure 5b. Predicted turbulence intensity along the minor axis of Mach 1.5 elliptic jet. 


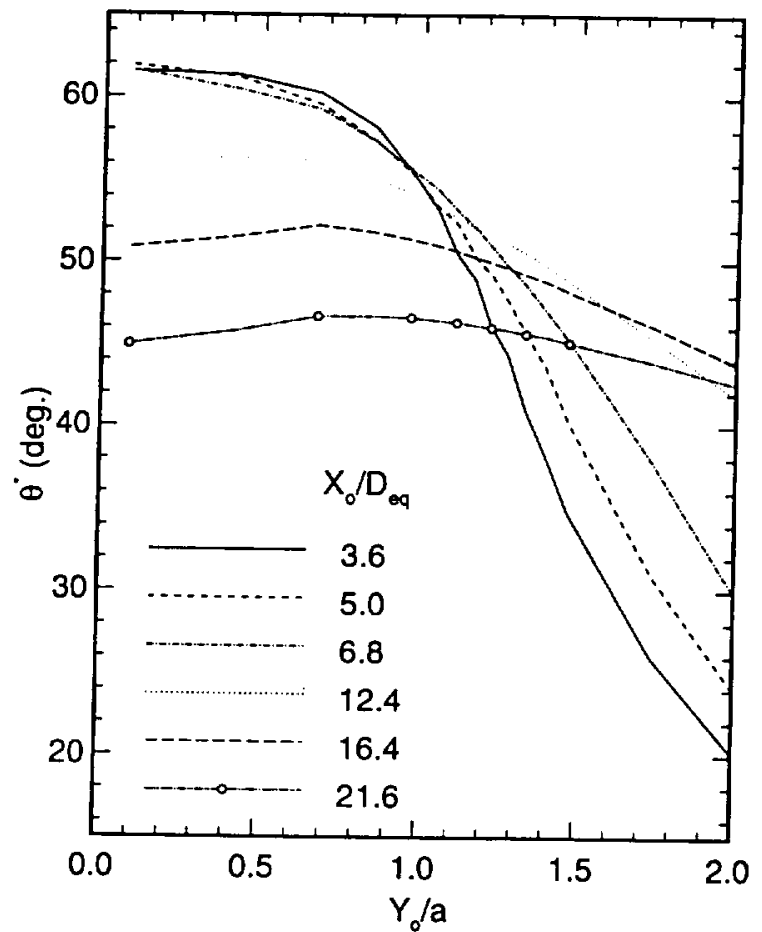

Figure 6 . Boundary of zone of silence vs. source location; $M=1.5, M_{\alpha}=0.0, A R=2: 1$ elliptic jet.

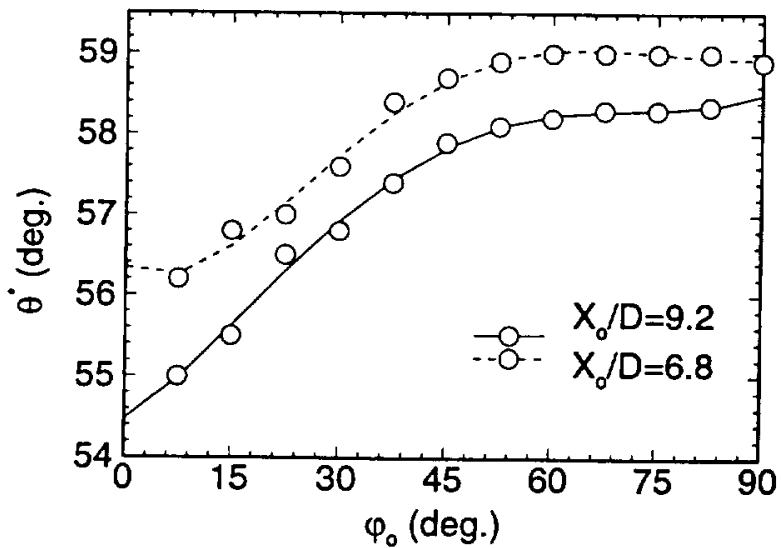

Figure 7. Boundary of zone of silence vs. azimuthal source location. Source at $\xi_{1} / a=1.0$.

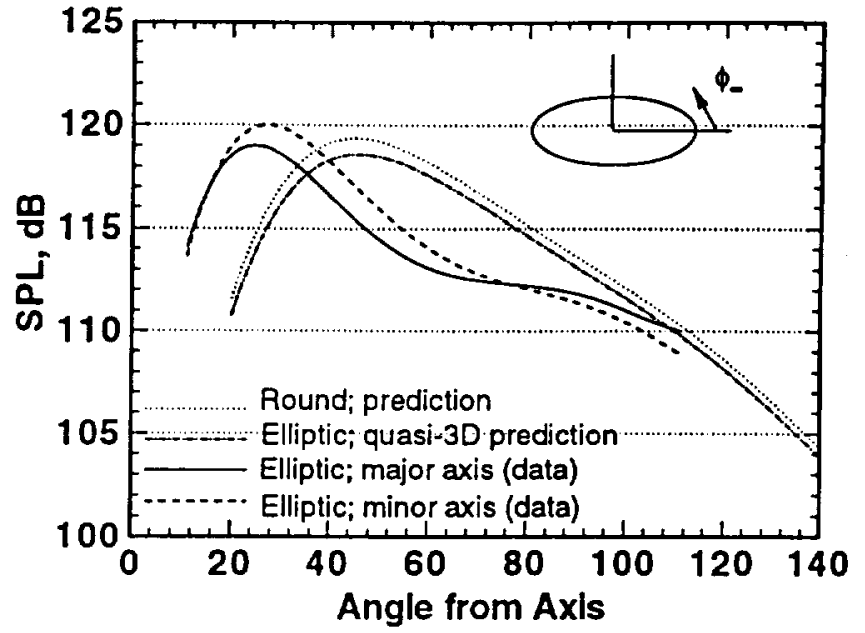

Figure 8. SPL directivity for Mach 1.5 round and elliptic jets.

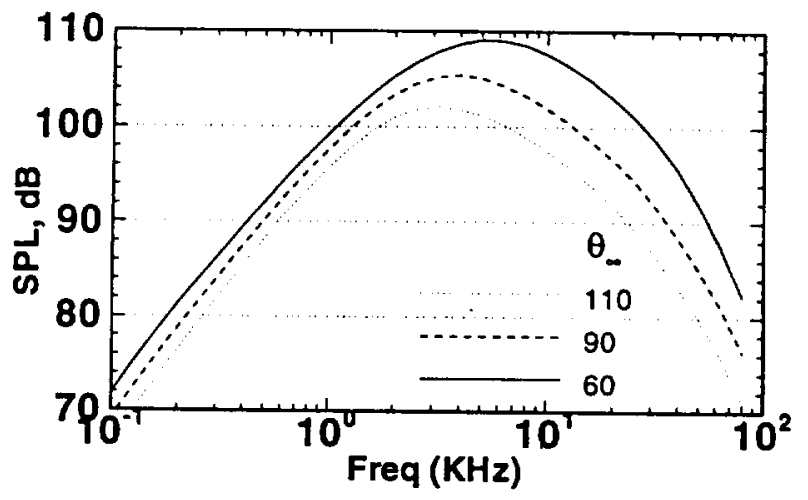

Figure 9. Predicted 1/3-Octave spectra; Mach 1.5 round jet.

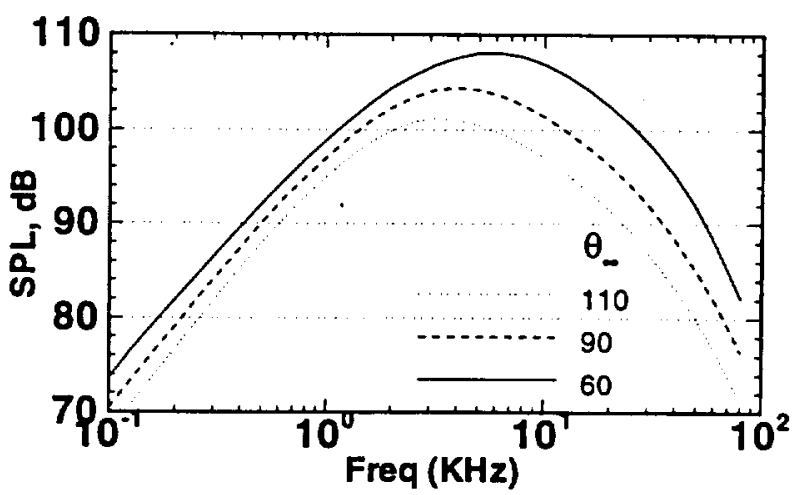

Figure 10. Predicted 1/3-Octave spectra; Mach 1.5. AR $=2 ; 1$ elliptic jet. (quasi-3D predictions) 


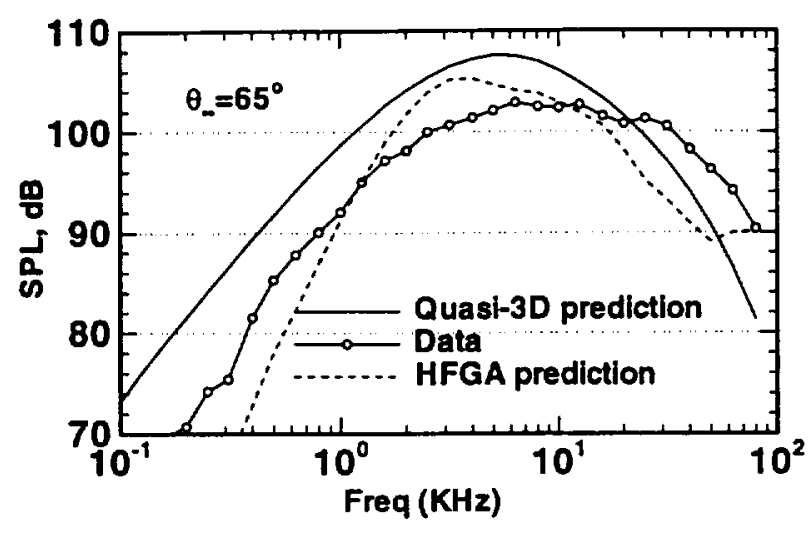

Figure 11a. 1/3-Octave spectra for Mach 1.5, $A R=2: 1$ elliptic jet at $\theta_{-}=65, \phi_{-}=0.0$.

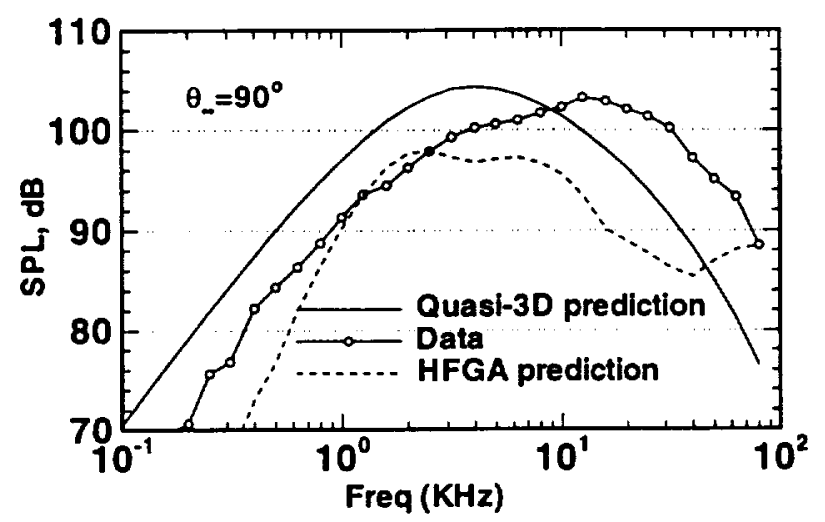

Figure 11b. 1/3-Octave spectra for Mach 1.5, $A R=2: 1$ elliptic jet at $\theta_{-}=90, \phi_{-}=0.0$.

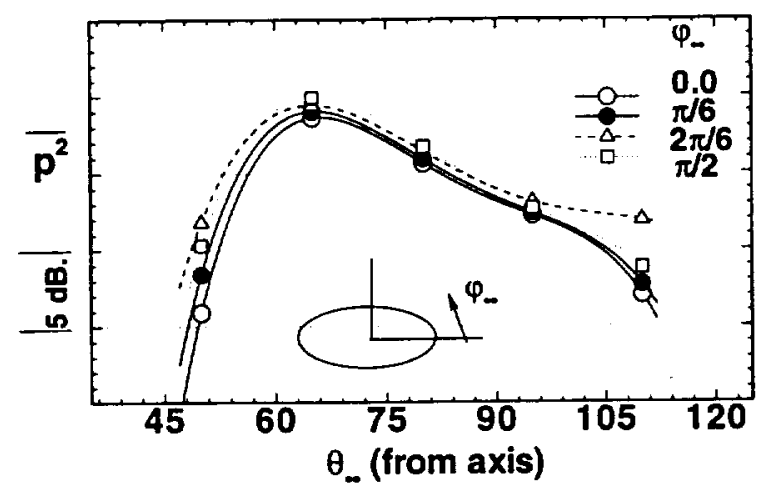

Figure 12. SPL directivity for Mach 1.5, AR=2:1 elliptic jet. (HFGA predictions)

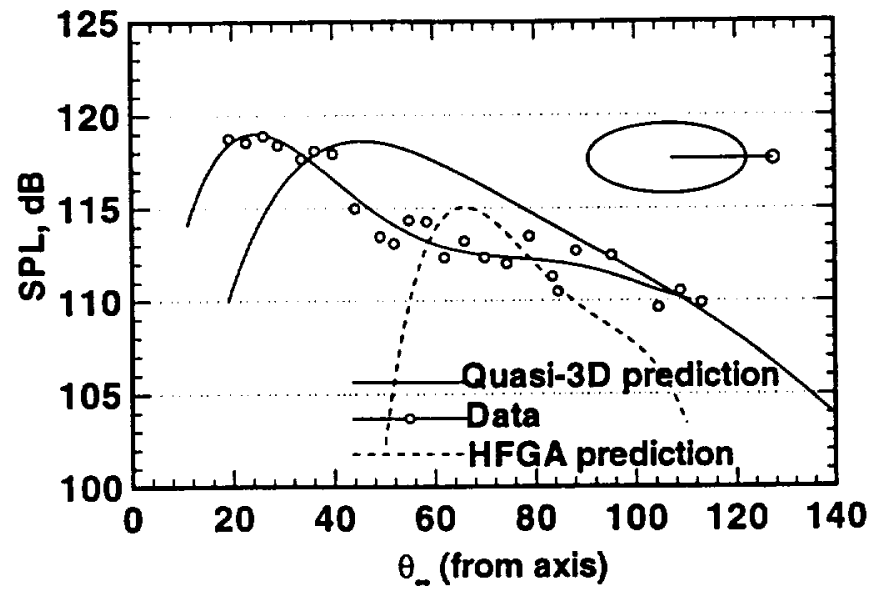

Figure 13. Comparison of SPL directivity; predictions vs. data for Mach 1.5 AR=2:1 elliptic jet.

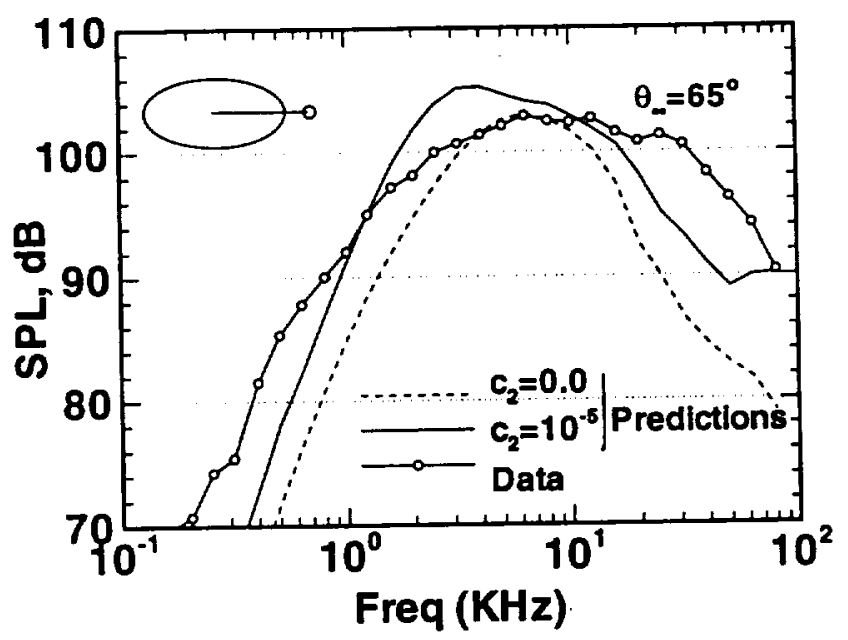

Figure 14. Predicted spectra for the elliptic jet (HFGA). Constant $c_{2}$ is defined in the modeling of the correlation function (Eq. 3).

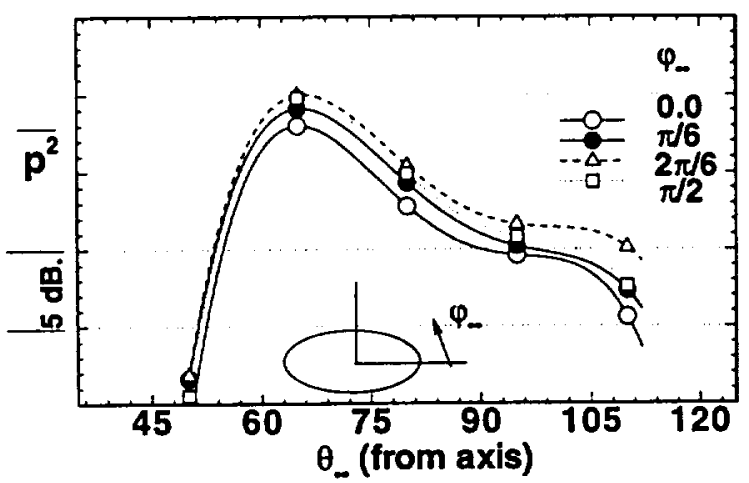

Figure 15. SPL directivity for a jet slice at $4.3<X / D_{\triangle Q}<5.8$, within Mach 1.5, AR =2:1 elliptic jet. (HFGA predictions) 



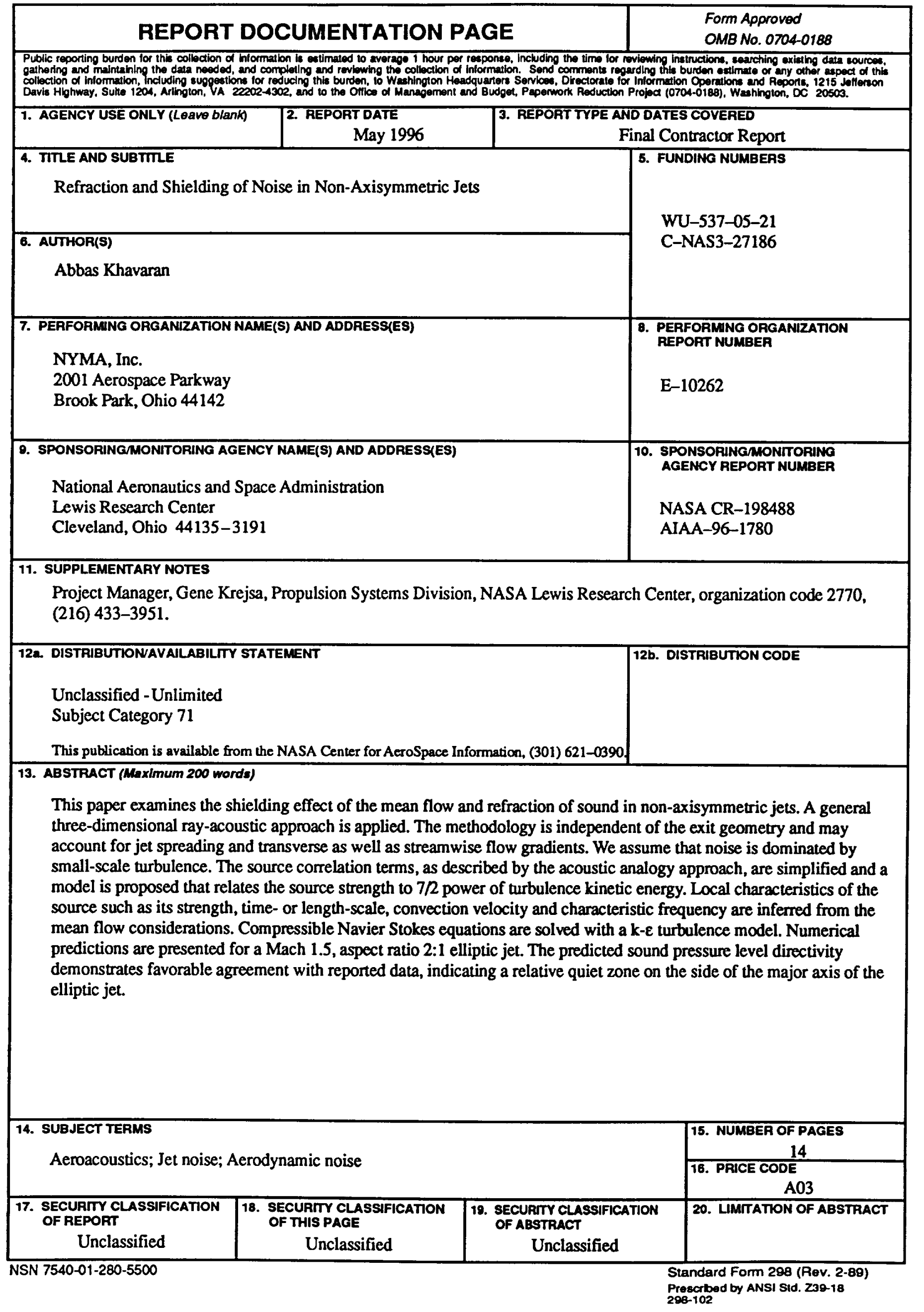





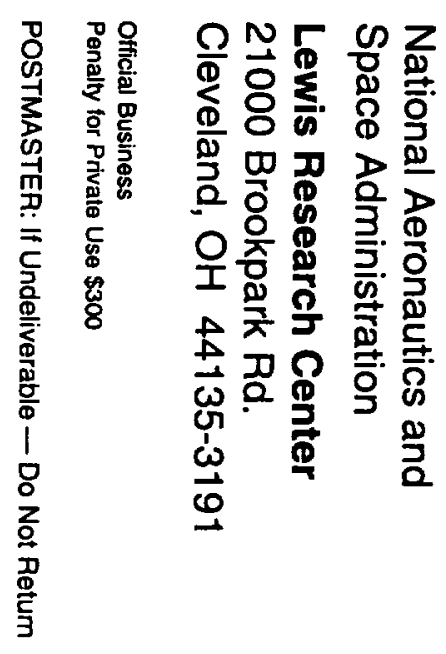

